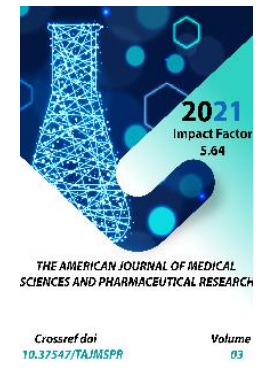

\title{
The Role Of The Nurse In The Prevention Of Bronchial Asthma In Children
}

Jamilakhon Erkinovna Kamilova

Assistant, Department Of Nurses With Higher Education At The Center For Advanced Training of Medical Workers, Uzbekistan

\section{ABSTRACT}

The relevance of the topic is determined by the increase in morbidity among the child population. Bronchial asthma is one of the most common childhood diseases, a formidable complication of which is asthmatic status, as well as the fact that the timely treatment of the disease, taking into account its severity and characteristics, the use of modern effective drugs are the key to the success of therapy, allow you to quickly stabilize the child's condition and further control bronchial asthma.

\section{KEYWORDS}

Bronchial asthma, endogenous allergens, exogenous allergens, immunoglobulins, dyspnea, orthopnea, peak flow meter, spacer

\section{INTRODUCTION}

Bronchial asthma in children is one of the most pressing medical and social problems. In the last decade, in the specialized medical literature, from year to year, the authors note 
an increase in the prevalence of bronchial asthma in the pediatric population and a tendency towards a more severe course of it.

The urgency of bronchial asthma in children is characterized not only by the transition of the disease to a chronic form, but also by the fact that the disease leads to an increase in disability among children, which negatively affects the resources of health care facilities.

Information on the increase in the incidence of asthma in recent decades has been published in a large number of foreign sources. For example, in the work of J.K. Fagan [23] and coauthors noted a high prevalence of diagnosed asthma (12.6\%) in the group of children from 7 to 12 years old.

In developed countries, 3-7\% suffer from bronchial asthma, in Central Asia - 1.4\%, in Tashkent $2.9 \%$ of the population per 1000 inhabitants. The most common infectiousallergic type is $85 \%$, the atopic type is $15 \%$ (From the study of A.M. Ubaydullaev).

If we talk about bronchial asthma, the causes of the disease should be distinguished separately. Because different factors can influence the development and progression of bronchial asthma in different regions of the world.

In particular, in Germany and in other Western and central foreign countries, along with malnutrition, other factors, such as stress, lack of physical activity, lead to an increase in chronic diseases in children, while in Uzbekistan, the main cause of bronchial asthma in children is bad ecology.

Referring to scientific articles and medical literature published by scientists, it becomes clear that the primary and general incidence of bronchial asthma among children (Fig. 1) and adolescents (Fig. 2) in the regions of Uzbekistan largely depends on the climatic conditions, the economic profile of the region and the level provision of the population with medical care.

\section{MATERIALS AND METHODS}

Indeed, in certain areas, such as the Khorezm and Navoi regions, the heat is much more dangerous, since the so-called effect of the heat islands is revealed. Heat and dust can cause severe asthma attacks. Summer comes, and with it comes heat, stuffiness, pollen of flowering grasses literally hangs in the air, and it is no longer up to summer joys for those who are irritated by this pollen.

Since the development of bronchial asthma in children is most often based on allergies, various allergens primarily affect the onset of an illness in a baby. The most common negative factors that provoke illness are industrial and chemical allergens, a polluted environment.

\section{RESULT AND DISCUSSION}

Studies show (from the study of K.Ch. Nurmamatova) the relationship between air pollution (an increase in the concentration of ozone, nitrogen oxides, acid aerosols and particulate matter) and a worsening of the course of bronchial asthma in children in theexample of Jizzakh, Navoin and Khorezm regions (Fig. 1 and fig. 2) 
Fig. 1

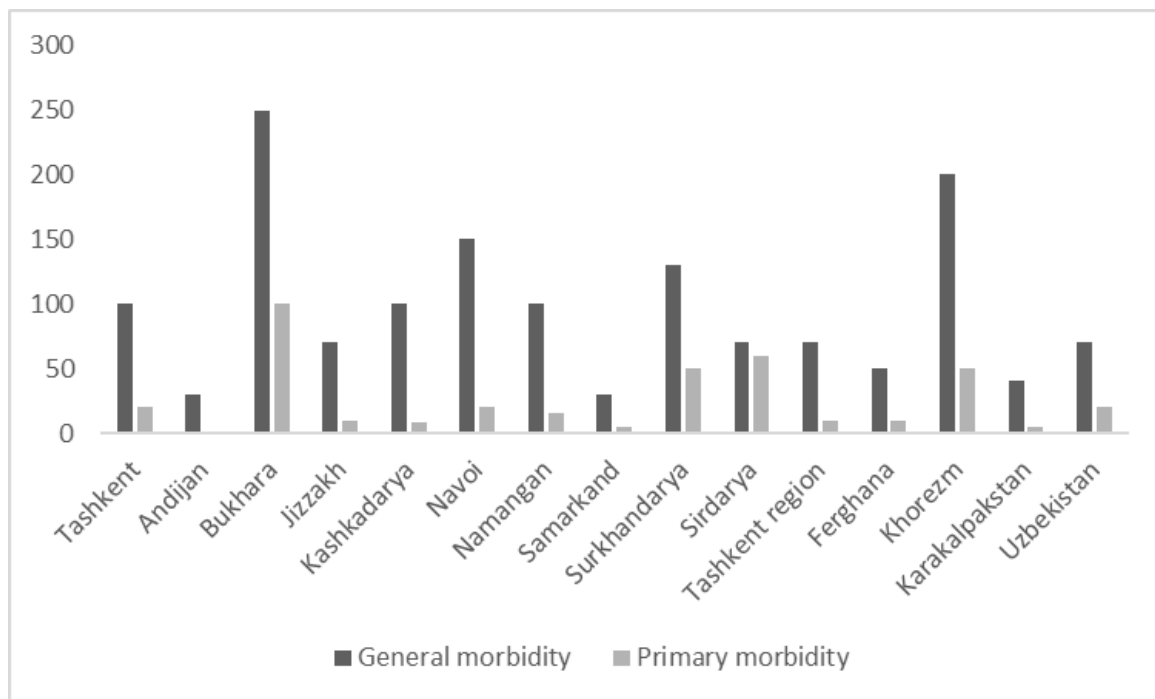

Fig.2

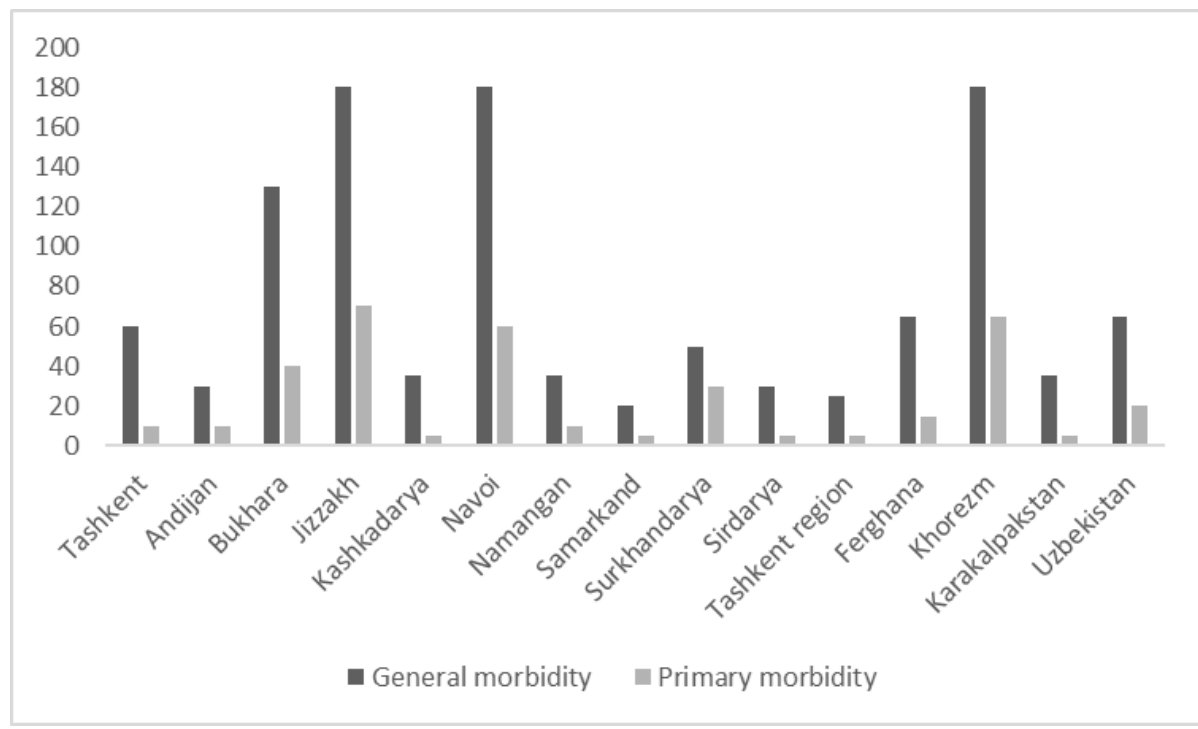

There are two main forms of bronchial asthma:

1. Exogenous (atopic, allergic) bronchial asthma, in the pathogenesis of which the production of specific antibodies related to immunoglobulins $\mathrm{E}$ (IgE) against common external (exogenous) allergens plays a key role. This form of bronchial asthma is diagnosed in $90 \%$ of asthma patients under 16 years of age.

2. Endogenous (non-atopic, non-allergic) form of bronchial asthma, in the genesis of which it is not possible to establish an allergic reaction to external (exogenous) factors (allergens). In this form of bronchial asthma, respiratory infections, impaired metabolism of arachidonic acid, endocrine and neuropsychiatric 
disorders, occupational factors, and volatile semiconductors of a non-allergic nature can serve as a triggering agent.

Exposure to various exogenous allergens, among which house dust becomes the most common risk factor, becomes a decisive factor in the development of $A D$ in most children. Wool, dandruff, saliva of animals (cats, dogs, guinea pigs, hamsters and other rodents), dry food for aquarium fish, as well as insects, especially cockroaches, can also provoke the development of bronchial asthma; mold spores; pollen from flowering plants; medications, especially antibiotics.

With prolonged exposure, dust affects the immune system - it reduces the activity of Tlymphocytes, inhibits the synthesis of antibodies of the main classes, stimulates the synthesis of immunoglobulins $\mathrm{E}$. increases the activity of the vagus nerve. In addition to the above factors, exacerbation of bronchial asthma can cause physical exertion, emotional stress, changes in the meteorological situation, as well as viral infections.

Sick children with bronchial asthma are worried about symptoms such as choking or shortness of breath, cough, shallow breathing, wheezing, orthopnea posture.

As the disease progresses in the body, such disorders occur as: general weakness, cyanosis of the skin, difficulty breathing, shortness of breath, dry cough, heart palpitations, dizziness and headache, susceptibility to various diseases.

\section{Nursing process in bronchial asthma}

The course and degree of achievement of control of bronchial asthma are determined by not only the volume and quality of the prescribed pharmacotherapy, but also by a number of non-drug factors. In achieving the required level of awareness, a significant role is assigned to nursing staff. The correctness of their actions directly determines the effectiveness of training, medical rehabilitation and secondary prevention in patients with bronchial asthma.

To date, there are no statistical data on the role of nurses in the treatment of children with bronchial asthma in Uzbekistan. Nevertheless, there is generally known information on which one can rely and draw a conclusion about the importance of nurses in the introduction of sick children with bronchial asthma.

In the world of medicine, there are many examples of unprecedented nursing care for patients with bronchial asthma. Many families with a sick child have repeatedly stated that they spend more time of the day with nurses than with doctors. In some Western countries, the duties of certain groups of nurses are very close to those of doctors. Nurses are closer to the patient than the doctor is.

How is the nursing asthma care plan implemented?

The role of a nurse in ensuring a normal lifestyle for children with asthma is invaluable, multifaceted and consists in therapeutic and diagnostic activities: the use of medications that are taken as prescribed by a doctor, use a peakfluometer, a nebulizer.

The nurse teaches the patient and his family how to use a pocket inhaler and issues of emergency first aid at the time of paroxysm, and gives recommendations regarding dietary nutrition, teaches how to behave after an attack.

A nurse has a clear structure, containing such stages as collecting information, identifying 
the problems of a sick child, planning and implementing patient care in a hospital.

In addition, the nurse must provide the patient or his parents with the following information:

- $\quad$ The cause of the disease, symptoms and aspects of the risk of bronchial asthma;

- The concept of allergens and antiallergic regimen;

- $\quad$ Basic drugs and their mechanisms of action;

- $\quad$ Types of inhalers;

- $\quad$ The procedure for using inhalers;

- The child's daily routine, physical activity, nutrition.

Actually, the work of a nurse with patients with bronchial asthma occurs in several stages:

1. Informs the patient. The nurse should talk with the patient's parents or with the child himself about the order in which people with asthma should live. If necessary, must provide the necessary literature. May recommend watching educational films such as Living with Asthma.

\begin{tabular}{|l|l|l|l|l|l|l|l|l|l|l|l|l|}
\hline Height & 109 & 109 & 109 & 109 & 109 & 109 & 109 & 109 & 109 & 109 & 109 & 109 \\
\hline PSV & 147 & 147 & 147 & 147 & 147 & 147 & 147 & 147 & 147 & 147 & 147 & 147 \\
\hline Height & 140 & 140 & 140 & 140 & 140 & 140 & 140 & 140 & 140 & 140 & 140 & 140 \\
\hline PS & 307 & 307 & 307 & 307 & 307 & 307 & 307 & 307 & 307 & 307 & 307 & 307 \\
\hline
\end{tabular}

4. Teaches to stop attacks of suffocation. Inhalation therapy helps to eliminate respiratory spasm, get rid of suffocation and normalize the child's well-being. The nurse's role is to choose the inhaler and how to use it correctly. Spacers are most suitable for children.
2. Teaches the correct breathing technique. Correct breathing is paramount in preventing new attacks of suffocation.

3. Teaches you to exercise self-control over your condition and use a peak flow meter.

A peak flow meter is a device for measuring the maximum (peak) expiratory flow (PSV). The modern device is convenient and easy to use; with its help, the patient can independently control his condition, calculate the dosage of the drugs taken and prevent an attack of suffocation. As a rule, children can use the device from 4-6 years old. The patient should independently or with the help of parents conduct a study twice a day, and enter all the peak flowmeter indicators into his schedule, with the help of which it is possible to better understand the features of the course of bronchitis and asthma.

To determine the degree of compliance of PSV with the norm, determine the proper value of PSV using tables taking into account the height of the child
The spacer is more likely not an inhaler, but an auxiliary device for inhalers with a dispenser; however, it should be singled out as a

separate group. Even if the baby did not breathe when pressing the bottle, you can be sure that the right dose will enter the respiratory tract. One of the primary responsibilities of a nurse is to teach the 
correct use of spacers, as inappropriate use of inhalers can lead to inappropriate dose distribution and economic costs.

5. Teaches how to behave after an attack.

Children often have a moist cough for a while after an attack. You can help sputum to stand out: with warm drink, taking mucolytics (Mukaltin, Ambroxol, Bromhexin), inhalation with bronchodilators (for example, with Ventolin). If tachycardia appears due to an attack, it is better to seek qualified medical help. It is important to warn the baby's parents that self-medication with beta-blockers can cause a new attack.

6. Gives advice on dietary nutrition and exercise.

Physical exercises with their therapeutic use, reflexively and humorally stimulating the respiratory centers, contribute to the improvement of ventilation and gas exchange. Under the influence of physical therapy, the general tone increases and the neuropsychic state of the patient improves; the central nervous system is toned; improved nervous processes in the cerebral cortex and the interaction of the cortex and subcortical centers; the body's defenses are activated.

The nurse also conducts a conversation with the patient or his parents about the features of a hypoallergenic diet, the expediency of its strict adherence. It is important to educate sick children that certain food restrictions do not mean that they will not be able to fully meet their food needs. His food is still sufficient and varied.

7. Emphasize the need to follow medical recommendations and explain to the patient that she should see a doctor in the following cases:

- With exacerbation of asthma symptoms;
- With shortness of breath, tightness in the chest or increased suffocation;

- When the inhaler does not help as before;

- With a decrease in the high speed of exhalation.

\section{CONCLUSION}

Summing up the article, I would like to note that a nurse must act professionally, quickly and accurately perform nursing interventions, since sometimes not only the health, but also the life of the little patient depends on this. Throughout her career, she will help relieve pain in children with bronchial asthma. This is the peculiarity of the nursing process in asthma.

The main conclusion that can be drawn is that the nursing process is a method of evidencebased and enforced practice of the nurse to provide care to patients. A nurse is not only an assistant to the attending physician, but also a kind of educator and moral support for patients.

\section{REFERENCES}

1. Mazurin A.V. "General care of children" / A.V. Mazurin, A.M. Zaprudnov, K.I. Grigoriev. - M :, Medicine 1998.

2. Belevsky, A.S. Interaction of a doctor and a nurse in the management of a patient with bronchial asthma / A.S. Belevsky // Atmosphere. Pulmonology and Allergology. - 2008. - No. 2.

3. Balabolkin I.I., Bulgakova V.A. Bronchial asthma in children. - $M$.: LLC "Publishing House" Medical Information Agency ", 2015.

4. Nurmamatova, K. Ch. The main indicators of the incidence of bronchial asthma in children in the Republic of Uzbekistan / K. Ch. Nurmamatova. - 
The American Journal of Medical Sciences and Pharmaceutical Research

(ISSN - 2689-1026)

Published: May 20, 2021 | Pages: 34-40

IMPACT FACTOR

2021: 5.64

Doi: https://doi.org/10.37547/TAJMSPR/Volume03Issue05-06

OCLC - 1121105510

Text: direct // Young scientist. - 2020. -

No. 17

5. Vershinina, Druk, Ratynskaya:

Bronchial asthma: Modern approaches to diagnosis and treatment: Textbook.

Phoenix, 2007

6. MedPortal.ru: Medical portal, everything about human health https://medportal.ru

7. https: //medvestnik.ru/ 